\title{
Use of Particle Agglutination Assay in the Diagnosis of Japanese Encephalitis and Dengue
}

\author{
Basu Dev Pandey ${ }^{1}$, Ramesh Pun ${ }^{2}$ and Krishna Prasad Pant ${ }^{3}$ \\ ${ }^{1}$ Sukraraj Tropical and Infectious Diseases Hospital, Kathmandu, Nepal \\ ${ }^{2}$ Nepal Academy of Science and Technology, Khumaltar, Lalitpur \\ ${ }^{3}$ Siddhanath Science Campus, Mahendranagar, Kanchanpur \\ e-mail:basupandey@wlink.com.np
}

\begin{abstract}
Japanese encephalitis (JE) and dengue are vector borne viral diseases that are endemic in the territory of Nepal. The purpose of the study was to assess the disease burden and to compare the results between particle agglutination and enzyme linked immunosorbent assay. A total of 185 serum samples of suspected Japanese encephalitis (JE) viral fever, dengue fever, malaria and typhoid were collected in the year 2005 (August-October) and 2006 (AugustNovember) from hospitals of Bardiya, Banke, Dang, Kathmandu and Parsa. The samples were investigated by particle agglutination and enzyme immunoassay at Everest International Clinic and Research Centre, Kathmandu. Out of 141 samples from suspected diseased patients, 51\% had a positive Japanese encephalitis virus (JEV) specific immunoglobulin $\mathrm{M}$ by particle agglutination assay while the anti-dengue immunoglobulin $\mathrm{M}$ positivity rate was $22.7 \%$ among 44 samples by the same assay. The specificity of particle agglutination kit against Japanese encephalitis and dengue was high as evident from absence of cross reactivity with other diseases like malaria, typhoid, kala-azar and leptospira. Thus, particle agglutination assay can be used as a tool for diagnosis of the diseases in developing countries like Nepal.
\end{abstract}

Key words: dengue virus, endemic, Japanese encephalitis, particle agglutination

\section{Introduction}

Japanese encephalitis (JE) is a mosquito borne infection caused by Japanese encephalitis virus (JEV) which is a member of Flavivirus (Flaviviridae). The virus is transmitted in an enzootic cycle between Culex mosquitoes and amplifying vertebrate hosts, primarily pigs and wading birds (Solomon et al. 2000). Primarily Culex tritaeniorhynchus is the principal vector. Humans accidentally acquire this infection by mosquito bites only when they encroach upon this enzootic cycle but JEV infected persons do not have high titre viraemia and are therefore considered as dead end hosts (Solomon 2003). The majority of human infections with JEV are asymptomatic and only about one in 250 infected people develop clinical symptoms (CDC 2008). JE is one of the most important causes of viral encephalitis worldwide with an estimated 50,000 cases and 15,000 deaths annually (Solomon et al. 2000). It is a serious public health problem prevalent predominantly in the southern Terai of Nepal for the past two decades. JE cases normally appear during April-May and reach at peak during late August to early September and decline from October (EDCD 2001). The numbers of cases have been increasing and the disease is expanding to new areas of Nepal every year. Twenty four districts of Terai and Inner Terai regions are affected by JE (EDCD 2008, Joshi 1983), and 1000-3000 cases and 200-400 deaths (EDCD 2008) due to JE have been reported every year.

Dengue is another flavivirus and one of the major vector borne diseases in the tropics. There is limited information about the presence of dengue infection in Nepal. The first case of dengue was reported in August, 2004 from Chitwan, Nepal (Pandey et al. 2004), followed by Hetauda. The outbreak of 2006 was observed at Nepalgunj (Banke), Tulsipur (Dang) and Birgunj (Parsa) and other nine districts pose serious threat to Nepal. At present, diagnosis and management of JE, 
dengue, Chikunguniya and other infectious diseases in Nepal are based on patients' clinical symptoms due to lack of diagnostic facility (Pandey et al. 2003). The series of previous studies using particle agglutination assay showed that the test was reliable and very simple which was useful in the developing countries. The present study was intended to compare the results between particle agglutination and enzyme linked immunosorbent assay and also to observe cross reactions with malaria, leptospirosis and other infectious diseases.

\section{Methodology}

A total of 185 serum samples were collected from patients clinically suspected of JE, dengue fever, viral fever, malaria and typhoid from Terai region of Nepal namely Bardiya, Banke, Dang and Parsa districts bordering to India. A total 141 samples were assayed from the samples collected during August to October, 2005 (Table 1, 2) and 44 serum samples from August to November 2006. Samples were also collected from patients referred to Sukra Raj Tropical and Infectious Disease Hospital from different area of Terai (Table 3, 4). Demographic information of patients was recorded before collecting samples. Particle agglutination assay was performed in Everest International Clinic and Research Center (EICRC), Kathmandu and compared with IgM ELISA (Combo ELISA kit) in the Nepalese serum.

The blood samples (5 $\mathrm{ml}$ from adult and $3 \mathrm{ml}$ from children) were collected from each suspected cases in sterile, clean, dry and labeled test tubes. The collected blood in test tubes was allowed to clot at room temperature. Then the blood in the test tube was centrifuged at $5000 \mathrm{rpm}$ for $5 \mathrm{~min}$ and the serum was separated. The serum was then collected in vials and stored at $4^{\circ} \mathrm{C}$ for a week. After then, the samples were transported to EICRC maintaining reverse cold chain. Aliquots for ELISA and PA were prepared and stored at $2-8^{\circ} \mathrm{C}$ until tested.

Sera were tested for IgM with PA assay kit (Pentax, Japan). The diluted serum (1:100) was added to the wells and incubated for $30 \mathrm{~min}$ at room temperature. After the wells were washed, DV/JEV antigen-coated beads slurry was added and allowed to settle for an hour. The result was interpreted by observing beads agglutination pattern.
For IgM capture ELISA, diluted serum sample $(1: 100)$ was added to the assay plate that was precoated with anti-human IgM antibody and incubated at $37^{\circ} \mathrm{C}$ for $60 \mathrm{~min}$. Concurrently, mixture of enzyme-labelled anti-flavivirus monoclonal antibody and inactivated DV/JEV antigen was added to the assay plate after the residual serum was removed from the plate by washing. After further 60 min incubation at $37^{\circ} \mathrm{C}$, the assay plate was washed and tetramethylbenzidine (TMB) substrate was added. After $10 \mathrm{~min}$, the reaction was stopped by addition of $1 \mathrm{M}$ phosphoric acid; TMB became yellow which was the indication of the presence of anti-DV/JEV IgM antibodies.

\section{Results}

Out of 185 samples, 141 suspected of JE and viral fevers were tested for anti-dengue IgM by PA and Combo ELISA (Table 1). Besides, the findings of both assays were compared to each other (Table 2). The remaining 44 samples from clinically diagnosed patients (Table 4) were tested for anti-dengue IgM by dengue IgM-PA and JE IgM-PA (Table 3).

Table 1. JE test result

\begin{tabular}{l|l|l|l}
\hline Test & Positive (\%) & Negative (\%) & N \\
\hline PA & $72(51)$ & $69(49)$ & 141 \\
ELISA & $59(41.8)$ & $82(58.2)$ & 141 \\
\hline
\end{tabular}

Table 2. Comparison between PA and IgM ELISA assay for JE

\begin{tabular}{l|l|l}
\hline PA & ELISA & $\mathbf{n ( \% )}$ \\
\hline+ & - & $22(15.6)$ \\
- & + & $9(6.4)$ \\
+ & + & $50(35.5)$ \\
- & - & $60(42.6)$ \\
\hline
\end{tabular}

$+=$ Positive

- = Negative

Table 3. Dengue test result

\begin{tabular}{l|l|l}
\hline Test & $\mathbf{N}$ & $\mathbf{( \% )}$ \\
\hline Dengue IgM PA & 10 & 22.7 \\
JE IgM PA & 27 & 61.4 \\
Both Positive & 9 & 20.4 \\
Both Negative & 16 & 36.4 \\
\hline
\end{tabular}


Basu Dev Pandey et al./Use of Particle Agglutination......

Table 4. Clinical diagnosis and application of PA

\begin{tabular}{l|l|l|l}
\hline Clinical diagnosis & $\mathbf{n}$ & IgM-PA for Dengue (\%) & IgM-PAfor JE (\%) \\
\hline Dengue fever & 7 & $7(15.9)$ & $6(13.6)$ \\
Japanese encephalitis & 25 & $1(2.3)$ & $19(43.2)$ \\
Viral fever & 8 & $2(4.5)$ & $2(4.5)$ \\
Kala-azar & 1 & Negative & Negative \\
Typhoid & 2 & Negative & Negative \\
Leptospira & 1 & Negative & Negative \\
Total & $\mathbf{4 4}$ & $\mathbf{1 0 ( 2 2 . 7 )}$ & $\mathbf{2 7}(\mathbf{6 1 . 4 )}$ \\
\hline
\end{tabular}

\section{Discussion}

PA showed higher (51\%) positivity rate than that of ELISA (41.8\%) for JE and viral fever suspected patients. Twenty-two (15.6\%) patients, those showed positive PA reaction were negative by ELISA while the number of patients positive by ELISA but negative by PA were only 9 (6.4\%). PA demonstrated superior specificity as compared to ELISA and the result was similar to the study done by Pandey et al. (2003).

Forty-four samples were collected during dengue outbreak in the year 2006. The patients were clinically diagnosed as having dengue fever, JE, viral fever, kalaazar, typhoid and leptospira. These patients were tested for anti-dengue IgM and anti-JEV IgM by PA. Among 44 patients, 22 were clinically diagnosed as having dengue fever and all were IgM positive to dengue, however almost (6) showed cross reactivity to JE. Similarly, 25 patients suspected of JE were mostly positive (19) to IgM-PA while cross reactivity to dengue was very minimal (1). The patients suspected of kala-azar, typhoid, leptospira were negative to both dengue and JE PA reaction. Overall, 22.7\% of patients showed positivity to dengue IgM-PA reaction.

The specificity of PA kit against dengue and JE was high as evident from absence of cross-reactivity with other diseases like malaria, typhoid, kala-azar and leptospira. The efficeiency of PA assay compared to IgM ELISA was significantly high. There is no much reactivity in a suspected case of JE by dengue infection. However, suspected cases of dengue tested by IgM-PA dengue kit showed some reactivity with IgM-PA JE. Therefore, PA assay is easier and more rapid in the diagnosis of JE and dengue in developing countries as compared to ELISA. This assay can be made available in every health centre because of its cost effectiveness and can be performed in simple laboratory settings as no sophisticated equipment is required.

It is evident that dengue outbreak has been established in different Terai regions of Nepal. However, the places from hilly region like Kathmandu valley is also at risk of contracting dengue disease because the vector Aedes aegypti responsible for transmission of the disease has been reported from urban areas of Basundhara and Gongabu-Balaju of Kathmandu District (Gautam et al. 2009). The availability of the vector might be due to the effect of global warming. So, earlier preparedness should be made to control outbreak of dengue in the future.

The limitation of the study is that the sample size was small so more serum samples from suspected dengue and JE patients should be collected for further validation of PA kit in Nepal. Moreover, ELISA result was interpreted through visual observation which might affect the result to some extent. Therefore, PA is a reliable tool for use in disease diagnosis and management. So, it can be used for the diagnosis of dengue and JE where the laboratory facility is not much pronounced.

\section{Acknowledgements}

We would like to thank all the patients, medical officers and laboratory technicians of different hospitals as well as staff of EICRC.

\section{References}

Center for Disease Control and prevention (CDC). 2008. Yellow book chapter-4. www.cdc.gov.ncidod. 
Epidemiology and Disease Control Division (EDCD). 2001. Annual report 2001. Department of Health Services (DoHS), Ministry of Health, Government of Nepal. Pp. 42-55.

Epidemiology and Disease Control Division (EDCD). 2008. Annual report 2006/2007. Department of Health Services (DoHS), Ministry of Health, Government of Nepal.

Gautam, I., M.N. Dhimal, S.R. Shrestha and S.R. Tamrakar. 2009. First record of Aedes aegypti (L.) vector of dengue virus from Kathmandu, Nepal. Journal of Natural History Museum 24:154-164.

Joshi, D.D. 1983. Incidence of Japanese encephalitis in children: 1978, 1979 and1980 outbreaks. Journal of Nepal Pediatric Society 2:18-25.
Pandey, B.D., S.K. Rai, K. Morita and I. Kurane. 2004. First case of dengue in Nepal. Journal of Nepal Medical College 6:157-159.

Pandey, B.D., A. Yamamoto, K. Morita, Y. Kurosawa, S.K. Rai.and S. Adhikari. 2003. Serodiagnosis of Japanese encephalitis among Nepalese patients by particle agglutination assay. Epidemiology Infection 131:881-885

Solomon, T., N.M. Dung, R. Kneen, M. Gainsborough, D.W. Vaughn and V.T. Khanh. 2000. Neurological aspects of tropical disease: Japanese encephalitis. Journal of Neurology Neurosurgical Psychiatry 68:405-415.

Solomon, T. 2003. Recent advances in Japanese encephalitis. Journal of Neurological Virology 9:274-283. 